\title{
The Optimization Concept of Service Fulfillment Measurement: A Research on Surgery Care
}

\author{
Norshahrizan Nordin ${ }^{1,2^{*}}$, Razli Che Razak ${ }^{3}$, Sharmini Abdullah ${ }^{4}$, Ummi Naiemah Saraih ${ }^{1}$, \\ Marniati $^{5}$ \& Wan Norsyafawati W. Muhamad Radzi ${ }^{1}$ \\ ${ }^{1}$ School of Business Innovation and Technopreneurship (PPIPT), Universiti Malaysia Perlis \\ (UniMAP), Kangar, Perlis, Malaysia \\ ${ }^{2}$ Center of Excellence Geopolymer \& Green Technology (CeGeoGTech), School of Material \\ Engineering, Universiti Malaysia Perlis (UniMAP), Perlis, Malaysia \\ ${ }^{3}$ Faculty of Business and Entrepreneurship, Universiti Malaysia Kelantan (UMK), PengkalanChepa, \\ Kelantan, Malaysia \\ ${ }^{4}$ Centre of International Languages, Universiti Malaysia Perlis (UniMAP), Pauh Putra, Perlis, \\ Malaysia \\ ${ }^{5}$ Universitas Ubudiyah Indonesia, Jln Alue Naga Desa Tibang, Banda Aceh, Indonesia
}

\begin{abstract}
This paper presents an optimization concept of service fulfillment measurement. Service delivery is assumed free from constraint, while service fulfillment is expected to achieve by maximizing the customer preferences. As the constraints are considered, the customer preferences are developed in a form of new model to set the optimum level of variables. The concepts will be further improved for medium and large scale optimization, which capable to evaluate the customer requirement in Kano-QFD integration. The results were also validated based on Kano Model and Quality Function Deployment (QFD) is integrated for patient attribute and service attribute prioritization. The new non-linear KanoQFD service satisfaction model has been developed, tested and validated with Kano model to facilitate the analysis and decision making for better service delivery improvement.
\end{abstract}

\section{Introduction}

Healthcare organization is struggling to provide customer driven quality service. The key success of healthcare service is the ability to develop a comprehensive healthcare service and deliver the best service direct to customer as required. Besides, hospitals are classified into primary, secondary, and tertiary hospitals based on bed size. Tertiary hospital is the highest class distributed in Malaysia [6]. There were 98 hospitals without specialist, 80 single specialist hospitals, 94 secondary hospitals, and 83 tertiary hospitals [5]. In terms of functionality, $\mathrm{MOH}$ hospitals were classified into five types: State Hospitals, Major Specialist Hospitals, Non-Specialist Hospitals, and Special Medical Institutions, which were based on workload, number of inpatient beds, and scope of serviced rendered [3]. 
Excellent healthcare services delivered to patients would not necessarily result in excellent patient service fulfillment, meet the patient expectation, and gain high satisfaction level. Therefore, patient satisfaction is multifactorial and difficult to measure [4];[5]). Inversely, poor healthcare services might not result in high dissatisfaction [6]. All are due to the nature of uncertainty feedback, which can be translated as non-linear behavior [7] and possesses complex quadratic relationship [8].

Based on Health Facts 2014 by MOH, Planning Division, Health Informatics Centre, from 2013-2014, out of 355 hospitals, 141 public hospitals and 214 private hospitals had 14,033 beds [9]. However, in terms of bed size, out of total 43,437 beds, 39,728 beds were found in the public sector, which covered $91.46 \%$ of bed density in Malaysia. The higher percentage reflected the public hospital domination on acute care service in Malaysia. This had been in line with $75.07 \%$ of 28,949 total doctors in Malaysia placed in public hospitals and 11,697 doctors at private hospitals. Doctors and specialists are the backbone entities in national healthcare service delivery. As of 31 st December 2013, the total number of doctors in Malaysia was 46,916 where $74 \%$ were in public hospitals and $26 \%$ were in private hospitals. This above doctor ratio to population shows the levels of stress and pressure among doctors that may affect the consistency of professionalism in daily medical practice. This is also the main reason for complaints on service by doctors had been the highest $(22.16 \%)$ in public hospitals [10]; [9].

Healthcare service providers need to reassess their strategies to cope with more challenging task with respond to continuation of customer demand. The healthcare challenging tasks are discussed in details by [11]. As a healthcare service provider, systematic reprogrammed and renewed assessments are the most significant step forward to cope with customer demand uncertainties. This is the only way to repositioning themselves in future. This scenario is true elsewhere. For example, an empirical study by [12] have observed that in $1999,40 \%$ of respondents have rated the Singapore hospitals' service quality as poor or very poor, definitely below patients' expectations. These findings have taken seriously by hospitals and $80 \%$ of them have absorbed TQM philosophy and develop customer oriented strategy. To be competitive, an alternative customer prioritizing approach mainly incorporates the Quality Function Deployment (QFD) and Kano's Model is proposed conceptually. The new mechanism concept is hope to effectively address a complaint which not only satisfies the customer but also an opportunity to create positive experience with customers, building a healthier foundation, stronger brand value and avoiding legal penalties. It also provides fair balance information for decision making while facing constraints such as operational, legal, human resource and market pressures.

\footnotetext{
*Corresponding author: norshahrizan@unimap.edu.my
} 


\section{Kano-QFD Integration Model: A Surgery Care}

The fast solution is needed to initiate the positive improvement of complaint handling. The solution should be incorporated with detail mechanism to quantify the complaints prioritization and analyze the complaints in regards to complainant and healthcare constraint perspective. To author's knowledge and support by literatures, there are no single model to incorporated with. A new optimization decision model needs to be developed comprehensively to incorporate and unite the prioritizing and analyzing element in a model. QFD can be mainly utilized effectively in healthcare services in two ways: First, to analyze customer expectations and characteristics of competitive services, and second, to define the prioritization of technical design characteristic for a new service design. Besides, QFD has been proven to be the prominent technique to resolve the marginal of uncertain customer requirement in a more effective way [13]. The ability to optimize and to analyze customer requirements in deciding the best service to be offered in advance is absolutely great in QFD. QFD and Kano model is found the best in its categories. Unfortunately, both are well success in product development but not in service sector as well as healthcare services [14]. In this paper, the new optimization decision model using QFD and Kano model is proposed and the related formulation in product development is replicated and modified to adapt with healthcare services.

The customer satisfaction coefficient indicates whether satisfaction can be increased by meeting requirement, or whether fulfilling this product requirement merely prevents the customer from being dissatisfaction [15]. The customer dissatisfaction coefficient indicates the other side [16].

Both can be expressed as:

Extent of satisfaction

$$
C S=\frac{f_{A}+f_{O}}{f_{A}+f_{O}+f_{M}+f_{I}}
$$

Extent of dissatisfaction

$$
D S=-\frac{f_{O}+f_{M}}{f_{A}+f_{O}+f_{M}+f_{I}}
$$

where $f_{A}, f_{O}, f_{M}, f_{I}$ represents the frequency of A,O,M,I respectively. The minus sign in Eq. 2 means that it is dissatisfaction. Eq.1 and Eq.2, CS and DS for each customer requirement can be calculated. 


\section{Methodological on Service Compliment (SCi) and Service Complaint Indexes (SCa)}

The index of $\mathrm{SCa}$ and $\mathrm{SCi}$ were inserted into QFD Step 2 according to defined PAs, orderly. For the case of Surgery Care, Fig. 1 shows the information of PAs, SCi and $\mathrm{SCa}$ that supply to QFD Phase 2 and 3.

Service Complaint (SCaKano SCaKano-ss) Index based on Service Dissatisfaction (SD)

Service Compliment (SCiKano-Q, $\mathbf{S C i} \boldsymbol{i}_{\text {Kano-SS }}$ ) Index based on Service Satisfaction (SS)

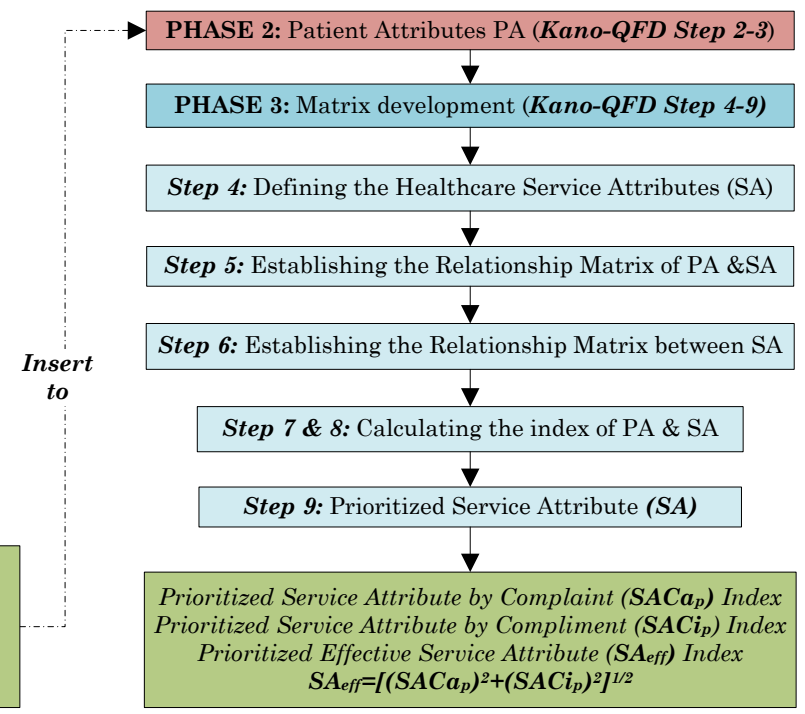

Fig.1. The flow of Kano-QFD execution for Phase 2 and Phase 3

\section{Results and Discussion}

Table 1. KQA Kano-SS statistical significant for Surgery Care

\begin{tabular}{ccccccccc}
\hline \multirow{2}{*}{ Variables } & & KQA & $\boldsymbol{a}$ & $\boldsymbol{b}$ & $|\boldsymbol{a}-\boldsymbol{b}|$ & $1.65 \sqrt{\frac{(a+b)(2 N-a-b)}{2 N}}$ & \multirow{2}{*}{ KQA significant? } \\
\hline & & & & & & & \\
& SCQ25 & M & 113 & 72 & 41 & $>$ & 18.66 & YES \\
& SCQ26 & M & 127 & 69 & 58 & $>$ & 18.96 & YES \\
& SCQ27 & M & 116 & 82 & 34 & $>$ & 19.00 & YES \\
& SCQ28 & M & 128 & 84 & 44 & $>$ & 19.32 & YES \\
& SCQ29 & M & 138 & 83 & 55 & $>$ & 19.50 & YES \\
& SCQ30 & M & 125 & 82 & 43 & $>$ & 19.21 & YES \\
& SCQ31 & M & 129 & 81 & 48 & $>$ & 19.28 & YES \\
& SCQ32 & M & 128 & 84 & 44 & $>$ & 19.32 & YES \\
& SCQ33 & M & 128 & 81 & 47 & $>$ & 19.26 & YES \\
\hline
\end{tabular}


Table 1 shows the $S C a$ and $S C i$ index for Surgery Care. It can be seen that index of "SCQ27: Flexible working hours" has the highest score of $S C i=0.38$. That means, in surgery, the time punctuality and management is experienced with the highest satisfaction. From complaints, the highest index is referred to "SQ26: Equipped room" where $S C a=$ 0.68 . It can be concluded, the most compliment service element is not necessarily the most complaint element, particularly for Surgery Care.

Table 2. Surgery Care Attributes Index

\begin{tabular}{ccccccccc}
\hline \multirow{2}{*}{$\begin{array}{c}\text { Surgery } \\
\text { Care }\end{array}$} & \multirow{2}{*}{ KQA } & \multicolumn{2}{c}{ Compliment (SS) } & \multirow{2}{*}{$\boldsymbol{S C i}$ Index } & \multicolumn{2}{c}{ Complaint (SD) } & \multirow{2}{*}{$\boldsymbol{S C a}$ Index } & $\boldsymbol{S}_{\text {eff }}$ \\
& & Kano-Q & Kano-SS & & Kano-Q & Kano-SS & & \\
\hline SCQ25 & M & 0.43 & 0.35 & 0.39 & -0.69 & -0.61 & -0.65 & 0.29 \\
SCQ26 & M & 0.43 & 0.24 & 0.335 & -0.69 & -0.56 & -0.63 & 0.25 \\
SCQ27 & I & 0.43 & 0.18 & 0.305 & -0.69 & -0.39 & -0.54 & 0.19 \\
SCQ28 & I & 0.43 & 0.16 & 0.295 & -0.69 & -0.38 & -0.54 & 0.19 \\
SCQ29 & M & 0.43 & 0.13 & 0.28 & -0.69 & -0.55 & -0.62 & 0.23 \\
SCQ30 & M & 0.43 & 0.17 & 0.3 & -0.69 & -0.52 & -0.61 & 0.23 \\
SCQ31 & M & 0.43 & 0.16 & 0.295 & -0.69 & -0.48 & -0.59 & 0.21 \\
SCQ32 & I & 0.43 & 0.12 & 0.275 & -0.69 & -0.5 & -0.60 & 0.21 \\
SCQ33 & M & 0.43 & 0.17 & 0.3 & -0.69 & -0.54 & -0.62 & 0.23 \\
\hline
\end{tabular}

Table 2 tabulates the list of Surgery Care elements (SCQ25-SCQ33) to represent the patient attributes (PA) that contains KQA frequency and Berger's coefficient. Most of the KQA frequency ranking trend follows of $\mathrm{M}>\mathrm{I}>\mathrm{A}>\mathrm{O}>\mathrm{Q}$ and only one SC element demonstrated $\mathrm{M}>\mathrm{O}>\mathrm{I}>\mathrm{A}>\mathrm{Q}$ but quality attribute $\mathrm{M}$ still dominated the KQA frequency. It can be observed that "SCQ26: Equipped room" encountered the highest SDss $=0.66$ and the highest SSss $=0.39$, that means patients have responded that SC element with high satisfaction impact even experienced the high dissatisfaction level. This scenario is against the scenario of Doctor Care and Nurse Care.

Table 3. Kano-SS KQA for Surgery Care

\begin{tabular}{|c|c|c|c|c|c|c|c|c|c|c|}
\hline \multirow{2}{*}{ Surgery Care } & \multicolumn{6}{|c|}{ Kano Quality Attributes (frequency) } & \multirow{2}{*}{$\sum$} & \multicolumn{2}{|c|}{ Berger's coefficient } & \multirow{2}{*}{ KQA } \\
\hline & A & M & $\mathrm{O}$ & I & $\mathrm{R}$ & Q & & $S S_{s s}$ & $S D_{s s}$ & \\
\hline SCQ25 & 50 & 113 & 65 & 72 & 0 & 0 & 300 & 0.38 & -0.59 & M \\
\hline SCQ26 & 47 & 127 & 69 & 56 & 0 & 1 & 300 & 0.39 & -0.66 & M \\
\hline SCQ27 & 55 & 116 & 43 & 82 & 0 & 4 & 300 & 0.33 & -0.54 & M \\
\hline SCQ28 & 47 & 128 & 37 & 84 & 0 & 4 & 300 & 0.28 & -0.56 & M \\
\hline SCQ29 & 35 & 138 & 35 & 83 & 0 & 9 & 300 & 0.24 & -0.59 & M \\
\hline SCQ30 & 47 & 125 & 44 & 82 & 0 & 2 & 300 & 0.31 & -0.57 & $\mathrm{M}$ \\
\hline SCQ31 & 45 & 129 & 41 & 81 & 0 & 4 & 300 & 0.29 & -0.57 & $\mathrm{M}$ \\
\hline SCQ32 & 50 & 128 & 35 & 84 & 0 & 3 & 300 & 0.29 & -0.55 & $\mathrm{M}$ \\
\hline SCQ33 & 45 & 128 & 42 & 81 & 0 & 4 & 300 & 0.29 & -0.57 & M \\
\hline
\end{tabular}


Logically, this particular condition happened due to the high frequency of quality attribute of One-dimensional $(\mathrm{O})$. Table 3 shows the frequency of $\mathrm{O}$ is higher than I and $\mathrm{A}$. The satisfaction impact of $O$ affects the Berger's coefficient value, significantly. Moreover, the lowest SDss is 0.54 which corresponds to "SCQ27: Flexible working hours". It refers the doctor and nurse that able and always ready for surgery and operation. The lowest SSss = 0.24 refers to "SCQ29: Easy appointment", that means the process of setting the surgery time and date is encountered some managerial problems.

Table 4. Kano-SS KQA and Berger's coefficient for Surgery Care

\begin{tabular}{|c|c|c|c|c|c|}
\hline \multirow{2}{*}{\multicolumn{2}{|c|}{ Service elements }} & \multicolumn{4}{|c|}{ Service gap } \\
\hline & & \multicolumn{2}{|c|}{ Pilot survey } & \multicolumn{2}{|c|}{ Mass survey } \\
\hline \multirow{9}{*}{  } & SCQ25 & -0.26 & $\mathrm{M}$ & -0.25 & M \\
\hline & SCQ26 & -0.32 & $\mathrm{M}$ & -0.29 & M \\
\hline & SCQ27 & -0.21 & I & -0.34 & M \\
\hline & SCQ28 & -0.22 & I & -0.21 & $\mathrm{M}$ \\
\hline & SCQ29 & -0.42 & $\mathrm{M}$ & -0.25 & $\mathrm{M}$ \\
\hline & SCQ30 & -0.35 & $\mathrm{M}$ & -0.27 & $\mathrm{M}$ \\
\hline & SCQ31 & -0.32 & $\mathrm{M}$ & -0.25 & M \\
\hline & SCQ32 & -0.38 & $\mathrm{M}$ & -0.23 & M \\
\hline & SCQ33 & -0.37 & $\mathrm{M}$ & -0.29 & $\mathrm{M}$ \\
\hline
\end{tabular}

Based on Table $4 \&$ Table 5 , the transition of KSS for Surgery Care is similar to Doctor Care and Nurse Care. In pilot survey, out of nine SCQ, only three SCQ are found significant (YES), but in mass survey, al the SCQ elements have resulted with significant (YES). SCQ33 has resulted with "YES" KQA statistical significant. This condition translates that all the SCQ is in normal distribution and the variation between first (a) and second (b) highest frequency is large enough to define the KQA for attribute M. The frequency difference $(\mathrm{a}-\mathrm{b})$ between attribute $\mathrm{M}$ and I is vary from $41-58$ which is higher than KSS coefficient $(18.66-19.50)$.

Table 5. Kano Statistical Significant for Surgery Care

\begin{tabular}{|c|c|c|c|c|c|c|c|c|}
\hline \multirow{2}{*}{ SV } & \multirow{2}{*}{ Codes } & \multicolumn{5}{|c|}{ Mass Survey } & & \multirow[b]{2}{*}{ KSS? } \\
\hline & & KQA & KSS? & KQA & & & & \\
\hline \multirow{9}{*}{ 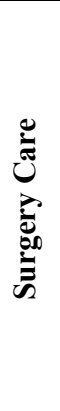 } & SCQ25 & $\mathrm{M}$ & YES & $\mathrm{M}$ & 41 & $>$ & 18.66 & YES \\
\hline & SCQ26 & M & NO & M & 58 & $>$ & 18.96 & YES \\
\hline & SCQ27 & I & NO & M & 34 & $>$ & 19.00 & YES \\
\hline & SCQ28 & I & YES & M & 44 & $>$ & 19.32 & YES \\
\hline & SCQ29 & M & NO & M & 55 & $>$ & 19.50 & YES \\
\hline & SCQ30 & M & NO & M & 43 & $>$ & 19.21 & YES \\
\hline & SCQ31 & M & NO & M & 48 & $>$ & 19.28 & YES \\
\hline & SCQ32 & M & NO & M & 44 & $>$ & 19.32 & YES \\
\hline & SCQ33 & $\mathrm{M}$ & YES & M & 47 & $>$ & 19.26 & YES \\
\hline
\end{tabular}


Table 6 shows the prioritized patient attributes (PACip) by compliments and (PACip) by complaints for Surgery Care. The "Hygiene rules and procedure" is found to be the highest complaints at $\mathrm{w}=78.72$ and compliments at $\mathrm{w}=50.43$ for Surgery Care. Similarly, the "Avoid unnecessary expenses" is shared for PACip and PACip as the lowest ranking. In terms of ranking order, both PACip and PACip listed in similar ranking order from rank 1 9.

Table 6. Prioritized patient attributes (PACip) by compliments for Surgery Care

\begin{tabular}{ccccc}
\hline Rank & $\%$ & w & Patient attributes (PA) & Codes \\
\hline 1 & 16.3 & 50.43 & Hygiene rules and procedure & SCQ25 \\
2 & 12.6 & 38.95 & Equipped room & SCQ26 \\
3 & 12.5 & 38.76 & Flexible working hours & SCQ27 \\
4 & 12.5 & 38.52 & Comfortable waiting room & SCQ33 \\
5 & 10.4 & 32.01 & Easy appointment & SCQ30 \\
6 & 10.3 & 31.68 & Very helpful staffs & SCQ31 \\
7 & 9.3 & 28.86 & Relaxing environment & SCQ30 \\
8 & 9.3 & 28.80 & Easy access location & SCQ32 \\
9 & 6.8 & 20.88 & Avoid unnecessary expenses & SCQ28 \\
\hline
\end{tabular}

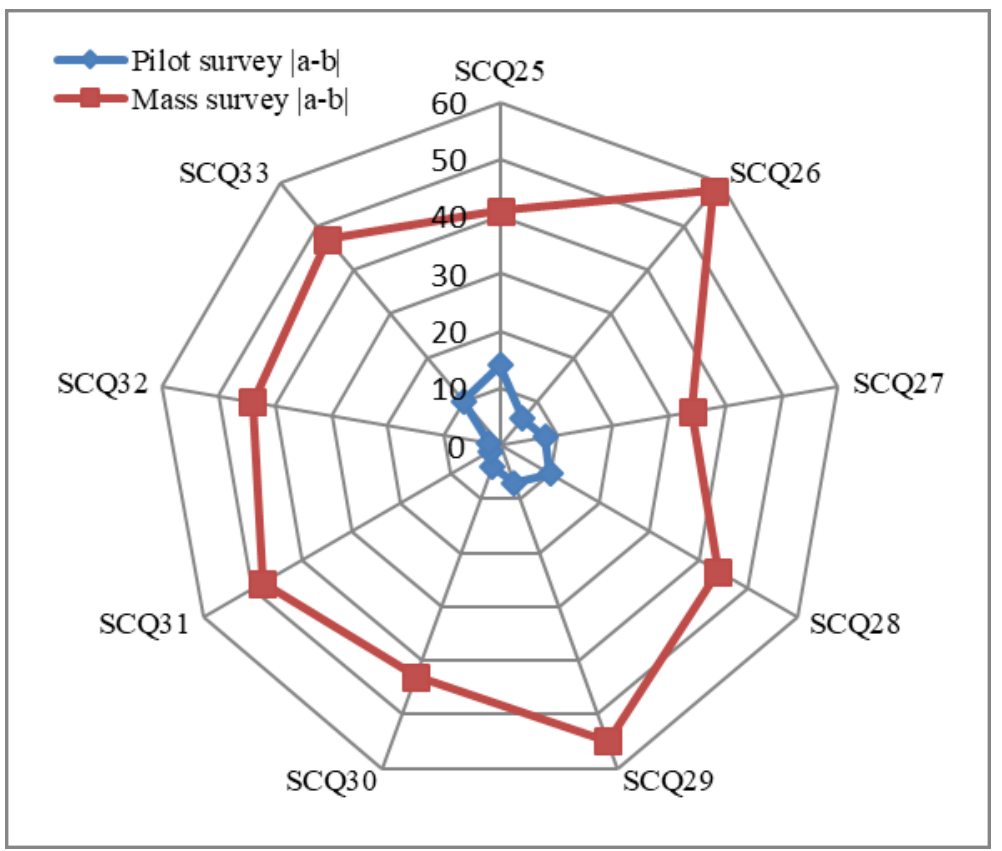

Fig.2. KSS spider chart map different $|a-b|$ for pilot and mass survey comparison based on first and second highest Surgery Care KQA closed frequency 
Fig.2 shows the KSS spider chart map different for pilot and mass survey comparison based on first $a$ and second $b$ highest frequency of Surgery Care KQA. It can be seen that most of insignificant KSS for pilot survey are laid below the value of 10 . To be significant, the value of $|a-b|$ must be over than 10. For mass survey, in order to be significant, the $|a-b|$ must over 18.66 at average but the figure shows the value of $|a-b|$ for all SCQ has lied over the value of 30. This result indicated the strong relationship along the SCQ elements based on KSS analysis.

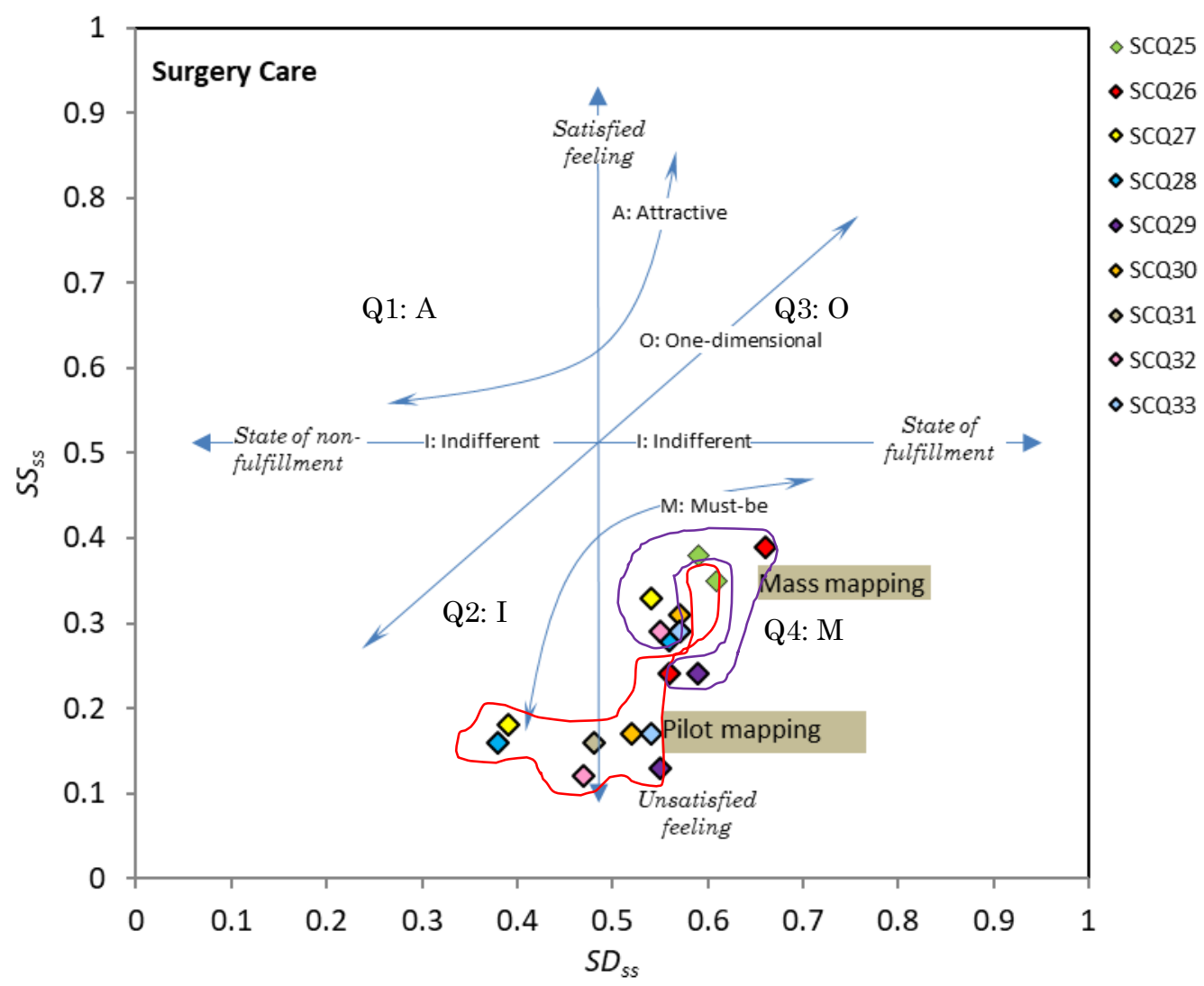

Fig.3. $S S_{\mathrm{ss}}-S D_{\mathrm{ss}}$ grid mapping for Kano-SS Surgery Care

Again, the composed and concentrated Must-be $M$ attribute has theoretically validated the present developed Kano-SS and Kano-Q in Kano-QFD integration by original Kano model. The similar scenario is happened to Surgery Care. Fig.3 shows SSss-SDss grid mapping for Kano-SS Surgery Care where the distribution of pilot mapping is scattered along the down side of unsatisfied feeling region. Most of the SCQ are placed in the Q2: I and near to the Unsatisfied feeling axis. Those attributes are marked as insignificant by KSS analysis. However, those attributes as placed well in upper region of Q1: M for mass mapping, where they have turned as significant and nearer to the state of fulfillment and lead towards satisfied feeling. That means, the proposed Kano-QFD satisfaction model suggests the HSP to improve the service attributes which were listed under "Medical Service Organization" and "Medical service delivery and technical care" in increasing the satisfaction level and reducing the level of dissatisfaction among patients. 


\section{Conclusions}

In service design aspect, the patient's responds can be accounted based on satisfaction levels from the context of patient feelings and fulfilment. The complaint and compliments mechanism is proved to reflect the dissatisfaction and satisfaction of patients [17]. The nonlinear relationship between patient attributes and service attributes has been successfully defined by Kano Quality Attributes (KQA) through Attractive (A), Must-be (M), Onedimensional (O), Indifferent (I) and Questionable (Q). The indexes of KQA translated the fulfilment of patients based on satisfaction and dissatisfaction [18]. The Kano-QFD integration provides the weightage between patient's attributes and service attributes and finally prioritised in ranking basis.

\section{Acknowledgments}

The authors are grateful for the research support of Ministry of Health (MOH) Malaysia and Universiti Malaysia Perlis (UniMAP) through awarded supportive facilities.

\section{References}

1. Onn, L. T., Sivasampu, S., \& Ariza, Z., National healthcare establishments and workforce statistics 2008-2009, Kuala Lumpur: Clinical Research Centre, Ministry of Health Malaysia, 1, 109 (2011)

2. Baba, N., Chong, T. S., Ibrahim, L., Salleh, A. R., Mokhtar, L. M., Ali, A.,Clinical Research Centre, Ministry of Health Malaysia 1 ed., 1, 119-130 (2011)

3. Merican, M. I., Ministry of Health Malaysia, ISSN 1511-1512 (2011)

4. Quaschning, K., Korner, M., \& Wirtz, M., Patient Education and Counseling, 91, 167$175(2013)$

5. Rowell, R. M., \& Polipnick, J., Journal of Manipulative and Physiological Therapeutics, vol. 31(8), pp. 602-610 (2008)

6. Driscoll, A., Currey, J., Marcia George OAM, R., \& Patricia M. Davidson RN, B., Australian Critical Care, 26, 55-57 (2013)

7. Lofgren, M., Witell, L., \& Gustafsson, A., The TQM Journal, 23(2), 235-246 (2011)

8. Meng, Q., \& Jiang, X., 2011 International Conference on Service System and Service Management (ICSSSM) (2011)

9. MOH, 2014 Health Indicators 2014, Planning Division, Health Informatics Centre (2014)

10. Baba, N., 2013 Retrieved Mac2013. http://www.pcb.gov.my/STATISTIK/Laman\%20Web\%20Statistik \%20AduanTahun\%202010\%20BM.pdf, 2011. 
11. Lim, P.C., \& Tang, N.K.H., Managing Service Quality, 10(2), 103-111 (2000)

12. Lim, P.C., \& Tang, N.K.H., International Journal of Healthcare Quality Assurance, 13(7), 290-299 (2000)

13. Chan, L. K. \& Wu, M. L., European Journal of Operational Research, 143, 463-497 (2002)

14. Chiou, C. C. \& Cheng, Y. S., IEEE International Conference on Industrial Engineering and Engineering Management (2008)

15. Nordin, N., \& Razak, R.C., Advanced Review on Scientific Research, 4(1), 2289-7887 (2014)

16. Lai, X., Xie, M. \& Tan, K. C., IEEE International Engineering Management Conference (2004)

17. Nordin, N., Abdullah., M.M.A.B, Mat, F., Abdullah, M., Razak, R.C., \& R., Adom, A.H., Assessment of Functional and Dysfunctional on Implant Stability Measurement for Quality of Life, MATEC Web of Conferences 97, 01116, (2017)

18. Nordin, N., Abdullah, M.M.A.B., Razak, R.C., Non-Linear Assessment and Deficiency of Linear Relationship for Healthcare Industry, AIP Conference Proceedings 1885, 020154 (2017) 